\title{
Blind Optical Modulation Format Identification From Physical Layer Characteristics
}

Eric J. Adles, Member, IEEE, Michael L. Dennis, Senior Member, IEEE, Wallace R. Johnson, Member, IEEE,

Timothy P. McKenna, Member, IEEE, Curtis R. Menyuk, Fellow, IEEE, Joseph E. Sluz, Senior Member, IEEE, Raymond M. Sova, Senior Member, IEEE, Michael G. Taylor, Member, IEEE, and Radha A. Venkat, Member, IEEE

\begin{abstract}
We describe and experimentally demonstrate automated identification of optical modulation formats exclusively from physical-layer signal characteristics. The system identifies the data encoding as on-off keyed versus binary or quadrature phase-shift keyed, as well as auxiliary modulation profiles. The symbol clock rate and optimal dispersion compensation are also determined. We have validated this system with over 1500 test cases. These are enabling functionalities for a universal optical receiver and for a cognitive optical network architecture that optimizes utilization of available optical bandwidth. We demonstrate the capabilities of the system using a wavelength division multiplexed signal consisting of 19 channels, with multiple modulation formats and widely varying dispersions, signal strengths, and optical signal-to-noise ratios.
\end{abstract}

Index Terms-Coherent communications, fiber optics communications, optical modulation.

\section{INTRODUCTION}

$\mathbf{T}$ HE recent development of optical communications receiver technologies based on coherent detection, combined with high speed digitization and digital signal processing (DSP) [1], [2], promises to revolutionize the field of optical communications. The approach enables the application of DSP techniques for optimization of the signal channel, including optimal matched filtering, compensation of linear signal impairments (chromatic and polarization mode dispersion), and potentially compensation of certain nonlinear impairments [3]-[6]. The technology also enables development of a universal optical receiver capable of demodulating a wide variety of optical data signals in the digital domain using a single set of optical and digitization hardware [7].

This latter capability is analogous to that of receivers for software-defined radio systems [8]. Similarly, it enables

Manuscript received September 4, 2013; revised January 21, 2014 and February 12, 2014; accepted February 19, 2014. Date of publication February 20, 2014; date of current version March 17, 2014.

E. J. Adles, M. L. Dennis, T. P. McKenna, J. E. Sluz, R. M. Sova, and R. A. Venkat are with the Johns Hopkins University Applied Physics Laboratory, Laurel, MD 20723 USA (e-mail: eric.adles@jhuapl.edu; michael. dennis@jhuapl.edu; timothy.mckenna@jhuapl.edu; joseph.sluz@jhuapl.edu; raymond.sova@jhuapl.edu; radha.venkat@jhuapl.edu).

W. R. Johnson is with the Virginia Polytechnic Institute and State University, Blacksburg, VA 24061 USA (e-mail: wajohnso@vt.edu).

C. R. Menyuk is with the University of Maryland Baltimore County, Baltimore, MD 21250 USA (e-mail: menyuk@umbc.edu).

M. G. Taylor is with Atlantic Sciences, Laurel, MD 20725 USA (e-mail: mtaylor@atlanticsciences.com).

Color versions of one or more of the figures in this paper are available online at http://ieeexplore.ieee.org.

Digital Object Identifier 10.1109/JLT.2014.2307555 cognitive optical networking (CON) architectures [9], analogous to cognitive RF networks [10], using on-the-fly allocation of data rates and modulation formats [11] negotiated between transmitter and receiver to optimize bandwidth utilization [12]-[14]. A key requirement for CONs is the ability to identify the data modulation format and any important transmission impairments directly from the received signals [8], [15]. At a network node, this capability serves the critical function of cueing optimal demodulation algorithms, and also serves as a line monitoring function for management of channel and bandwidth allocations within the optical domain [16].

In this paper, we describe and experimentally demonstrate blind identification of the modulation format for wavelength division multiplexed (WDM) signals across the C-band $(1530-1560 \mathrm{~nm})$. For each channel, the detected signal is analyzed to determine the symbol rate and to identify the optimal dispersion compensation. The modulation format is then identified from among fifteen possible combinations of data encoding, auxiliary modulation, and alternative implementation methods. We have verified that the format identification (FID) algorithms are tolerant to a wide range of optical signal-to-noise ratios (OSNRs from 9 to $>30 \mathrm{db} \cdot 0.1 \mathrm{~nm}$ ) and are principally limited by the analog bandwidth of the digitizer.

We note that the requirements for blind identification of modulation format are somewhat different from the requirements for a coherent receiver optimized for demodulating a specific format. As the clock rate is unknown a priori, oversampling is required to ensure that the signal samples satisfy the Nyquist criterion with respect to the maximum baud rate, whereas synchronous sampling of a single time per symbol is sufficient for demodulation of the signal data. Similarly, general purpose determination of the chromatic dispersion (CD) from the received signal will require being able to examine a broad range of values that may be different from case to case (e.g., in examining multiple channels within a WDM system), whereas an optimized receiver will, in normal operation, be set to compensate a single fixed dispersion value corresponding to the impairment of the individual channel. Likewise, blind determination of the clock rate may require analyzing over a broad frequency range (approximately a decade in this work), as opposed to the narrow-band clock recovery typical for a commercial receiver.

It is common in studies of communications systems to use many acronyms, and this work is no exception. For the convenience of the reader, we have included an acronym table and their meanings at the end of the paper. 


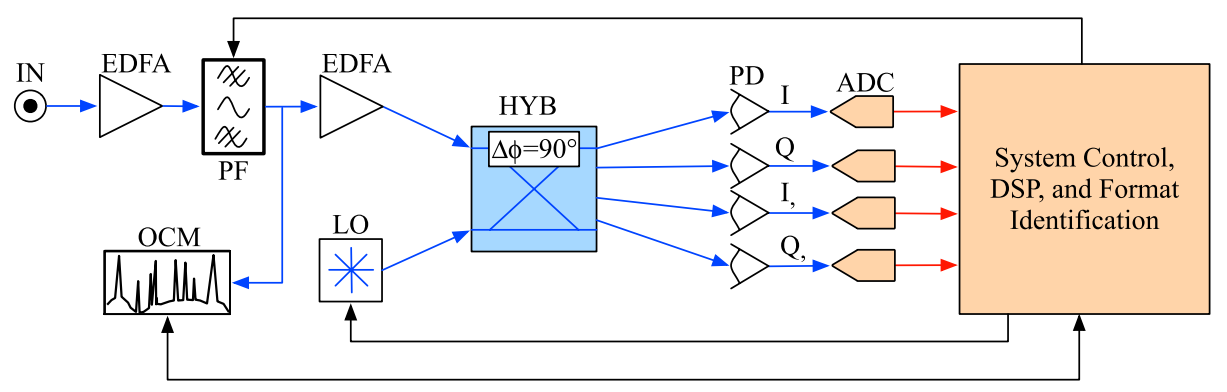

Fig. 1. Format identification system block diagram. IN-optical input, EDFA-erbium doped fiber amplifier, PF-programmable optical filter, OCM-optical channel monitor, LO-local oscillator, $\mathrm{HYB}-90^{\circ}$ optical hybrid, PD-photodiodes, ADC-analog to digital converters, DSP-digital signal processing.

\section{MOdUlATION FORMAT IDENTIFICATION SYSTEM}

Fig. 1 illustrates the FID system hardware in block diagram form. The hardware is polarization diverse, but in this paper we are only reporting results from single polarization signals. A computer manages the process and automates the processing of a set of channels. An input optical signal is preamplified and, with the programmable optical filter (PF) fully open, its spectrum is captured using an optical channel monitor (OCM). All channels reported by the OCM are then successively identified. For each channel, the PF is programmed with a user-defined passband $(10 \mathrm{GHz}-1 \mathrm{THz})$ centered on the identified channel. For the results presented here, a bandwidth of $40 \mathrm{GHz}$ was used, consistent with a $50 \mathrm{GHz}$ channel spacing. The signal is amplified to a preset power and fed into a coherent optical detection subsystem (Optametra OM4106) with a tunable local oscillator (LO). The LO is tuned to match the channel frequency $( \pm 2 \mathrm{GHz})$. The resulting in-phase and quadrature photocurrents for each polarization are digitized using a real-time digital oscilloscope (Tektronix DPO72004, 50 GSample/s, 20 GHz effective analog bandwidth). The identification algorithm can determine the format of a channel with as few as 40,000 points, corresponding to a capture time of $<1 \mu \mathrm{s}$. The digitized time series are $I / Q$ demodulated in software [1], [17] and passed to the identification algorithm.

The data encoding format is determined as either on-off keyed (OOK), binary phase-shift keyed (BPSK), or quadrature phase-shift keyed (QPSK). For formats without auxiliary amplitude modulation (AAM), the algorithm can distinguish between a primary or alternative implementation, viz., standard nonreturn-to-zero (NRZ) versus optical duobinary (ODB), BPSK implemented using a Mach-Zehnder modulator (MZM) versus a phase modulator (PM), and QPSK implemented using a dualparallel Mach-Zehnder (DPMZ) versus a MZM followed by a PM (MZPM). AAM is determined from among the principal implementations based on MZMs: $33 \%, 50 \%$ or $67 \%$ duty cycle return-to-zero pulses (RZ33, RZ50, RZ67). The algorithm is capable of distinguishing the combinations of encoding and auxiliary modulation as listed in Table I. Although not all are in common use, these constitute a wide variety of legacy modulation formats that have been implemented commercially. Advanced modulation formats, such as polarization multiplexed or quadrature amplitude modulation formats are not considered in this work due to limited access to appropriate transmitters.
TABLE I

OPTICAL MODULATION FORMATS

\begin{tabular}{|c|c|c|c|c|}
\hline \multirow{2}{*}{\multicolumn{2}{|c|}{ Aux. Mod. }} & \multicolumn{3}{|c|}{ Data Encoding } \\
\hline & & OOK & BPSK & QPSK \\
\hline \multirow{2}{*}{ None } & Primary & OOK NRZ & BPSK MZ & QPSK DPMZ \\
\hline & Alternate & OOK ODB & BPSK PM & QPSK MZPM \\
\hline \multirow{3}{*}{ RZ } & $33 \%$ & OOK RZ33 & BPSK RZ33 & QPSK RZ33 \\
\hline & $50 \%$ & OOK RZ50 & BPSK RZ50 & QPSK RZ50 \\
\hline & $67 \%$ & OOK RZ67 & BPSK RZ67 & QPSK RZ67 \\
\hline
\end{tabular}

Potential extensions of the algorithms to accommodate such formats will be discussed in Section IV.

The FID algorithm first accounts for imperfections in the acquisition system. Delay differences between the photodiodes and digitizers of the four channels are compensated by interpolating to a common time base. Amplitude, phase, and polarization errors in the optical hybrid are accounted for by multiplying the time series by a calibration matrix generated during manufacture. Fig. 2 shows a flowchart of the FID stages that determine the required dispersion compensation, clock rate, principal modulation format, and for PSK cases, whether the modulation is implemented with a MZM or a PM. The steps outlined in Fig. 2 will now be discussed in detail.

$\mathrm{CD}$ compensation and clock recovery are nested processes. The range of $\mathrm{CD}$ compensation values are defined as an array by the user. For our tests we used values in the range of -4500 to $+4500 \mathrm{ps} / \mathrm{nm}$ corresponding to the $\mathrm{CD}$ that we could generate with our laboratory equipment. The step size used within this range varied depending on the clock rate that was being searched as follows: $500 \mathrm{ps} / \mathrm{nm}$ for $<5.63 \mathrm{GHz}, 200 \mathrm{ps} / \mathrm{nm}$ for $5.63-$ $12.66 \mathrm{GHz}, 100 \mathrm{ps} / \mathrm{nm}$ for $12.66-18.98 \mathrm{GHz}$, and $50 \mathrm{ps} / \mathrm{nm}$ for $>18.98 \mathrm{GHz}$. The grid step was chosen to correspond to an accuracy (for \pm 1 grid step) such that negligible dispersion penalty would result for the corresponding dispersion compensation. The dispersion parameter $\gamma$ is defined

$$
\gamma=\beta_{2} L B^{2}=\frac{\lambda^{2}}{2 \pi c} \Delta(D L) B^{2}
$$

where $\beta_{2}$ is group velocity dispersion, $L$ is the length, and $B$ is the bandwidth. We take $\Delta(D L)$ to be the dispersion step size. For the selected step sizes $\gamma<0.05$ at the upper limit of each band. From Ref. [18] this would correspond to a maximum power penalty of $<0.5 \mathrm{~dB}$. The selection of these clock frequencies is discussed in the next paragraph. A finer grid spacing 


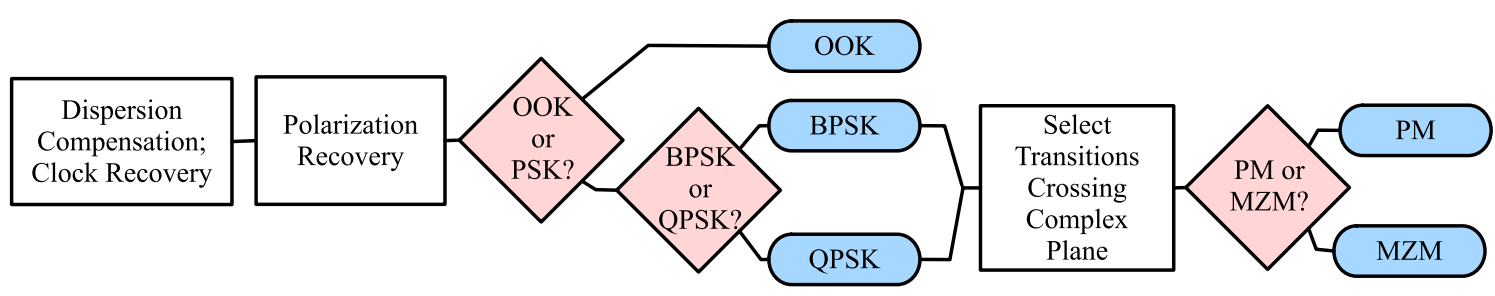

Fig. 2. Flowchart illustrating the steps for determining the required chromatic dispersion compensation, clock rate, modulation format, and, for PSK signals, implementation.
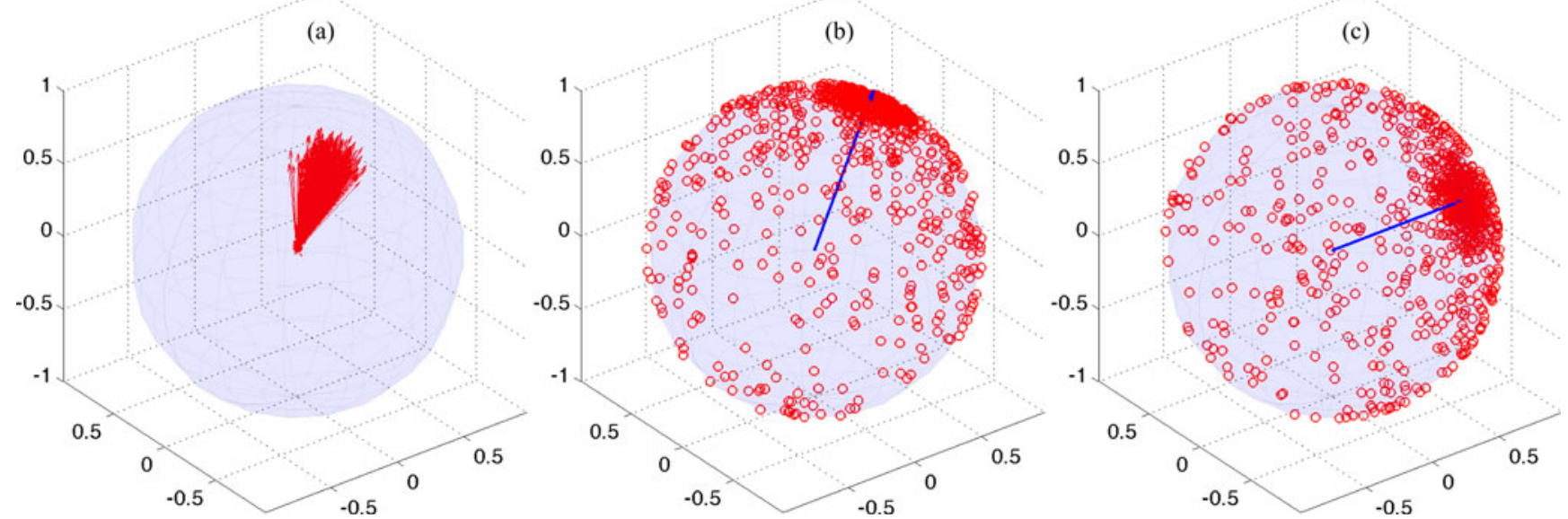

Fig. 3. Stokes vectors of symbol-centered data are shown as red arrows in (a). In (b) and (c) normalized Stokes vectors of symbol-centered data are shown as red circles on the surface of a unit sphere. The normalized vector sum of the Stokes vectors (average SOP) is represented as a blue arrow. The blue arrow indicates the received average SOP before (b) and after (c) rotating the SOP to lie along the $x$-axis.

will increase the accuracy of the $\mathrm{CD}$ algorithm at the expense of computation time. For each $\mathrm{CD}$ compensation value, a corresponding finite impulse response filter is applied to the time series, and then a clock search is performed.

Clock recovery occurs by searching for the peak frequency in six frequency bands: $2.5-3.75,3.75-5.63,5.63-8.44, \ldots$, 18.98-23 GHz. To eliminate the second and higher harmonics, the upper limit of each frequency band is equal to $1.5 \times$ the lower limit. The upper limit of $23 \mathrm{GHz}$ is consistent with the analog bandwidth of our digitizers. To eliminate harmonics of the clock rate, the data is low-pass-filtered to pass each band. Because for many common formats, including most formats without AAM, no clock signal is present in the received power or field signal, a quartic nonlinearity is applied and an FFT is performed. A coarse estimate of the clock frequency is made by picking the peak frequency within each clock band. This initial estimate is limited to the FFT resolution. For a sample rate $F_{s}=50 \mathrm{GSamples} / \mathrm{s}$, and record length $N=40,000 \mathrm{sam}-$ ples, the FFT resolution is $F_{s} / N=1.25 \mathrm{MHz}$. The accuracy of the clock estimation is improved to within $\pm 20 \mathrm{kHz}$ by a combination of spectral interpolation and parabolic fitting [19]. The magnitude of the peak frequency within each band is then stored as a figure of merit (FOM). A matrix of FOMs is generated over clock bands and $\mathrm{CD}$ compensation values. Certain OOK formats (e.g., NRZ, ODB) may exhibit local maxima near, but offset from, the optimal dispersion compensation value, so a smoothing function is applied to the curve of the clock power as a function of dispersion compensation to eliminate them.
The maximum of the smoothed FOM matrix indicates the required CD compensation and the clock rate. The compensation is applied, and the data is retimed to the symbol clock.

To recover the state of polarization (SOP), the symbolcentered data is transformed into a Stokes vector representation. The vector sum of the Stokes vectors then indicates the SOP of the received signal. From this calculated SOP, a rotation matrix is generated and applied to rotate the SOP to lie along the $x$ axis. Fig. 3 illustrates this process for a 10 GBaud NRZ signal. In Fig. 3(a), the Stokes vectors have been normalized to the largest magnitude. The vectors representing binary value 1 clearly indicate the as-received SOP, while vectors representing binary value 0 are randomly oriented around the origin. In Figs. 3(b)(c), the normalized Stokes vectors of symbol-centered data are shown as red circles on the surface of the Poincare sphere, and the normalized vector sum of the Stokes vectors (average SOP) is shown as a blue arrow. Fig. 3(b) shows the as-received SOP while (c) shows the average SOP after rotating it to lie along the $x$-axis. The same procedure is used for PSK signals. For PSK signals the SOP is more clear because the power is constant and the average SOP is slowly varying on the time scales we are observing.

With the polarization aligned, identification of the principal modulation format can proceed. To distinguish between OOK and PSK signals we look at the ratio $\sigma(|E|) / \mu(|E|)$, where $\sigma$ indicates the standard deviation, $\mu$ indicates the mean, and $|E|$ is the magnitude of the complex electric field. Since the histogram of $|E|$ is bimodal for OOK signals, we expect that 

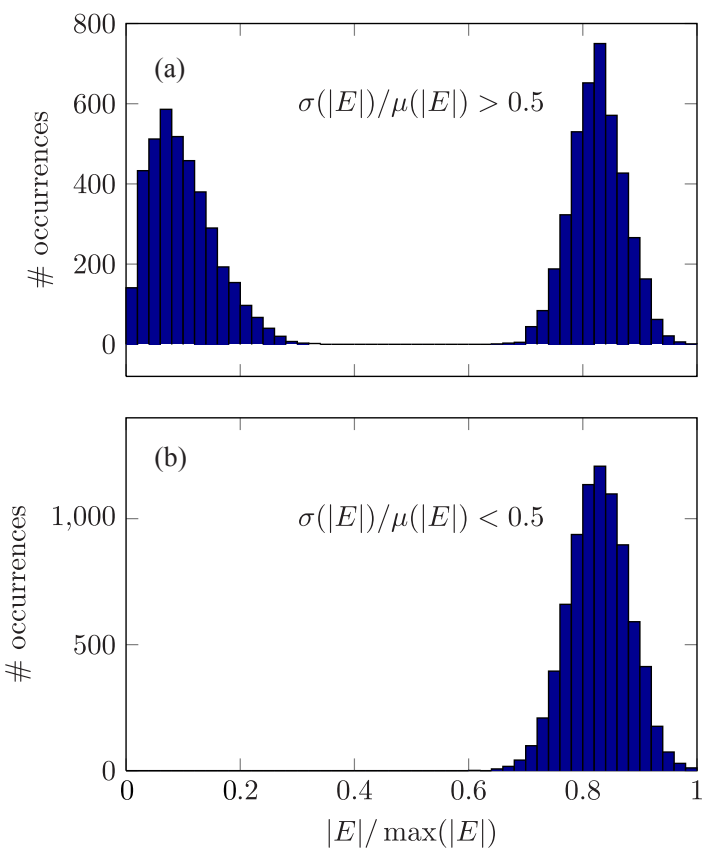

Fig. 4. Histogram of $|E|$ for (a) OOK and PSK (b) signals.

this ratio $\approx 1$. For PSK signals, the histogram of $|E|$ has a single mode. Therefore, we expect this ratio $\ll 1$ for PSK signals. The FID algorithm uses 0.5 as the decision limit for distinguishing between these two formats. This decision is illustrated in Fig. 4.

It is important to note that at this point, we have not accounted for the carrier-LO offset frequency $f_{\mathrm{CO}}$. Therefore, the recovered constellation will rotate at $f_{\mathrm{CO}}$. This frequency can be used to distinguish between BPSK and QPSK signals by considering differences in constellation symmetry between BPSK and QPSK signals. A BPSK signal has two clusters of constellation points opposite one another on the complex plane, that rotate at $f_{\mathrm{CO}}$. When the complex values corresponding to these points are squared the result is one cluster of points which rotates at $2 f_{\mathrm{CO}}$, and the Fourier transform has a peak at $2 f_{\mathrm{CO}}$. For a QPSK signal, the square results in two clusters of points that average to zero. Therefore, the Fourier transform of $E^{2}$ does not have a prominent peak for QPSK signals. When either a BPSK or QPSK signal is raised to the fourth power, $E^{4}$, the result is a single cluster rotating at $4 f_{\mathrm{CO}}$, and the Fourier transform has a prominent peak at that frequency. This difference can be seen in Figs. 5(a) and (b). We can distinguish between a BPSK and QPSK signal by

$$
\alpha=\max \left[\operatorname{FFT}\left(E^{2}\right)\right]^{2} / \max \left[\operatorname{FFT}\left(E^{4}\right)\right]
$$

where $\alpha \approx 1$ for BPSK signals and $\alpha \ll 1$ for QPSK signals. After the BPSK/QPSK decision is made, the data is adjusted to remove the carrier-LO offset.

AAMs are often applied to the data pulse profile to improve the overall signal transport characteristics, particularly in longhaul $(>600 \mathrm{~km})$ and ultra-long-haul $(>1,000 \mathrm{~km})$ systems. The FID algorithm is designed to distinguish the presence or absence of AAM. Furthermore, other information relevant to the physical signal can be determined, such as the method of implementation

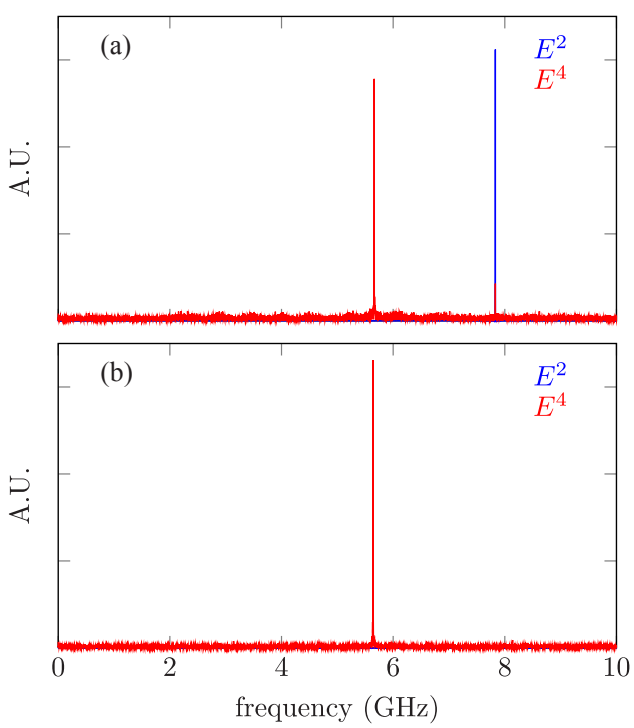

Fig. 5. Using the carrier offset frequency to determine whether a signal is (a) BPSK or (b) QPSK.

for signals without AAM, and the pulse profile for signals with AAM.

In practice, AAM is implemented in a variety of ways. We have focused on data that is modulated using three methods that are readily implemented using standard lithium niobate MZMs. The ideal implementations yield pulses with $33 \%, 50 \%$, and $67 \%$ duty cycle, and so are referred to in Table I as RZ33, RZ50 and RZ67. To identify the type of RZ modulation, we examine the interpolated time series covering symbol-to-symbol transitions, from the center of one symbol to the center of the next. Transitions between symbols of the same value, are identified, binned, and averaged. For BPSK signals this yields usable mean time series for the 1-1 transitions and for the $0-0$ transitions; for QPSK signals there are four such averaged time series, while only the 1-1 transition profile is relevant for OOK signals. The resultant time series is Fourier transformed, and the bandwidth of the result compared with the bandwidth expected for each of the three ideal pulse profiles convolved with the digitizer bandwidth. This method reliably identifies the RZ pulse profile at symbol rates up to $\approx 20 \mathrm{GHz}$, limited by the effective oscilloscope bandwidth of $20 \mathrm{GHz}$.

For signals with no AAM, ancillary information can be determined either from the symbol histograms or from the transitions. For OOK signals, standard NRZ is distinguished from ODB by calculating the mean field amplitude at symbol center: For NRZ, the mean will be $\approx 0.5$, while for ODB, the +1 and -1 symbols cancel yielding a mean of $\approx 0$. The two standard methods for implementing BPSK modulation can be distinguished via the average electric field at the mid-transition point between different symbols (i.e., 1-0 and 0-1): For an MZM-based implementation, the power passes through a null, while for a PM-based method, the power is constant. The two are thus distinguished by whether the normalized $\left\langle E_{\text {transition }}\right\rangle>0.05(\mathrm{PM})$ or $\left\langle E_{\text {transition }}\right\rangle<0.05$ (MZM). QPSK signals are most commonly generated by optical vector modulation using a DPMZ, 
although alternatives using a MZPM, or a PM driven with a multilevel signal can be envisioned. The DPMZ method is distinguished from alternatives according to the average power in transitions between symbols that are adjacent in the constellation.

\section{EXPERIMENT RESULTS AND DISCUSSION}

In order to determine the limitations of our approach, we have experimentally tested FID for all formats in Table I. For each format, a single-channel signal was generated at clock rates of 5, 10, 15, 20 and 23 Gbaud, with OSNRs of 9, 12, 15, 20 and $30 \mathrm{~dB} \cdot 0.1 \mathrm{~nm}$, and applied dispersions ranging from -4100 to $+1500 \mathrm{ps} / \mathrm{nm}$. Altogether, we examined a set of 1487 test cases. Thirty-one cases were discarded due to obvious configuration errors. Because the power in the clock tone is used to determine the $\mathrm{CD}$, and symbol-centered data is used for determining the format, a clock identification failure is the most consequential error for our algorithm. The clock rate was correctly determined in all but four cases, all of which were at $23 \mathrm{GBd}$. We note that this is in the rolloff region of the analog bandwidth and near the Nyquist limit of our digitizers. There were 16 cases where the clock rate was correctly identified but the $\mathrm{CD}$ was not identified to within 2 points of the $\mathrm{CD}$ search grid: 10 cases were at clock rates $\geq 20 \mathrm{GHz}$ which we attribute to limited digitizer bandwidth; and 6 cases were at OSNRs of 9-12 db·0.1 nm which we attribute to poor OSNR. The modulation format was correctly identified in all but 24 cases: 9 and 3 cases were at 23 and $20 \mathrm{GBd}$ respectively which we attribute to limited digitizer bandwith; 6, 4, and 2 cases were at 9,12 , and $15 \mathrm{~dB} \cdot 0.1 \mathrm{~nm}$ OSNR. From these results we conclude that digitizer bandwidth is the principle limitation of the technique with respect to oversampling, bandwidth, and impairments. Auxiliary modulation profiles are accurately identified up to limitations imposed by the digitizer bandwidth; the RZ duty cycle is difficult to distinguish at baud rates $>20 \mathrm{GHz}$. We first present some general results from this data set, and we then present results from a WDM experiment.

Fig. 6(a) illustrates the dependence of the clock error on the baud rate and on the number of analyzed points in the time series. Each point plots the mean absolute error, $\left\langle\left|R-R_{0}\right|\right\rangle$, where $R$ is the calculated baud rate, and $R_{0}$ is the actual baud rate, averaged over all formats and all OSNR values at the corresponding baud rate. Only those data sets that we collected with no dispersion were used for this calculation. Two important conclusions can be drawn from this plot: 1) short time series introduce a floor on the clock rate accuracy; 2) clock error increases with increasing baud rate.

It should be noted with respect to Fig. 6(a) that the standard time series length used for analysis in FID (40 000) yields a floor of $\approx 11 \mathrm{kHz}$. This is $\approx 100 \times$ higher accuracy than the simple Fourier transform limit, validating the usefulness of the two-stage refinement, and is more than adequate for accurate symbol timing and alignment. However, if higher precision is desired, Fig. 6(a) shows that it can be obtained by using a longer time series. In the test data, no floor is observed for time series of $\geq 128,000$ points.
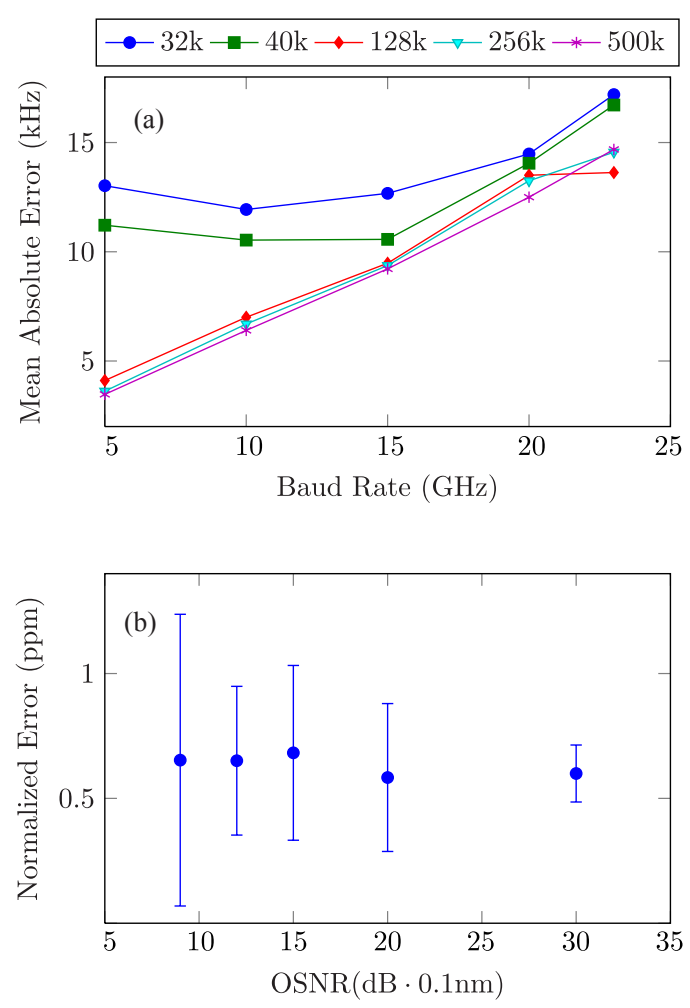

Fig. 6. (a) The mean clock error versus record length. (b) The normalized clock error versus OSNR.

We note that the increasing error with increasing baud rate is not due to the clock recovery algorithm, but rather reflects a frequency offset between the master clock used for the test data signals (an Anritsu frequency synthesizer) and the reference clock of the digitizer (i.e., the Tektronix 72004 oscilloscope). The limiting slope of the curves corresponds to a relative precision of $0.6 \mathrm{ppm}$, within what may be expected for laboratory test and measurement instrumentation.

The dependence of clock error on OSNR is shown in Fig. 6(b). To factor out the increase of error with baud rate noted above, we plot the mean normalized error $\left\langle\left(R-R_{0}\right) / R_{0}\right\rangle$. For Fig. 6(b), the maximum number of collected time series points (500 000) has been analyzed. We used the full record length to ensure that the limited precision of short time series will not dominate any effects due to OSNR. Fig. 6(b) shows that the calculated clock rate is not strongly dependent on the OSNR, even over the wide range of OSNRs tested. For all tested OSNRs, the magnitude of the mean normalized error is $\approx 0.6 \mathrm{ppm}$, corresponding to the slope for the 500000 point curve in Fig. 6(a). The positive sign of this error indicates a small frequency offset for the reference oscillator of the Tektronix oscilloscope with respect to that for the pattern generator, with the former being faster than the latter. As might be expected, there is a general decrease in the statistical clock error, as indicated by the length of the error bars, with improving OSNR. At high OSNR, the results indicate that the overall statistical error in the clock determination can be as low as $0.1 \mathrm{ppm}-1 \mathrm{kHz}$ for a 10 GBaud signal-for a time series of $10 \mu \mathrm{s}$. We note that this corresponds to an improvement of $\approx 100 \times$ compared with the Fourier transform limit, as described above. 

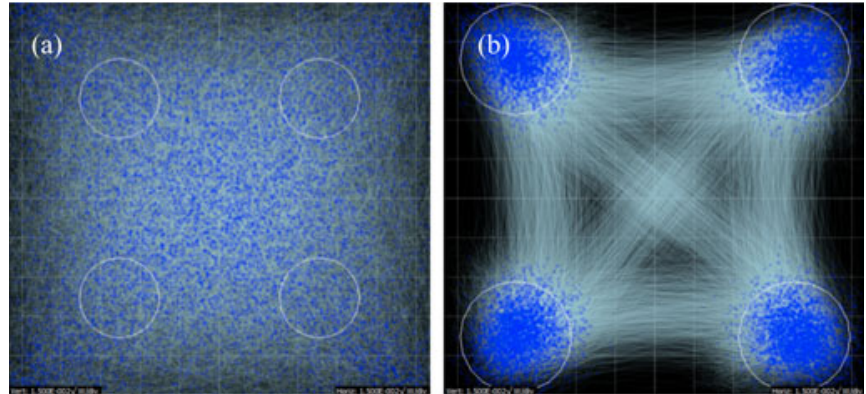

Fig. 7. Experimental results showing the constellation of a 21.509 GBaud QPSK signal $(43.018 \mathrm{~Gb} / \mathrm{s})$ with $-4100 \mathrm{ps} / \mathrm{nm}$ dispersion (a) before and (b) after digital CD compensation.

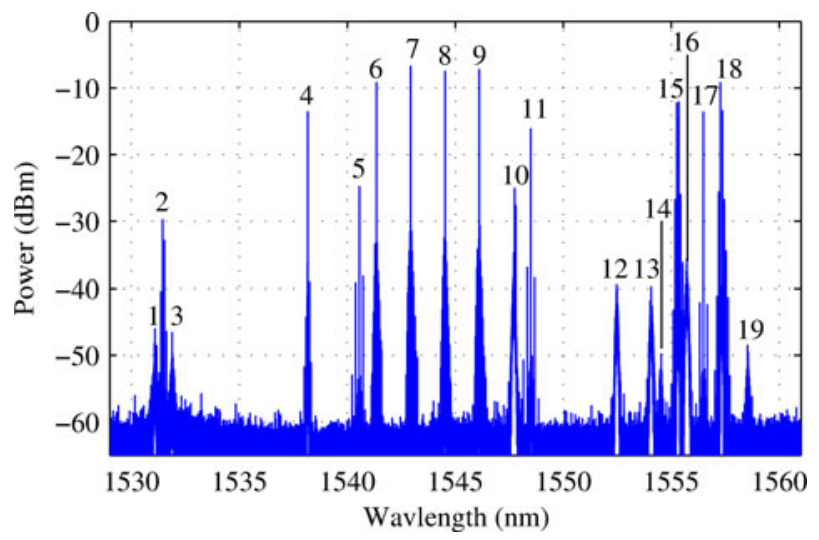

Fig. 8. $10 \mathrm{MHz}$ resolution optical spectrum of test WDM signal plan. This plan includes OOK, BPSK and QPSK signals with data rates ranging from 4.25 to $42.67 \mathrm{~Gb} / \mathrm{s}$, dispersion ranging from -2600 to $+800 \mathrm{ps} / \mathrm{nm}$ and OSNRs of 13 to $>30 \mathrm{~dB} \cdot 0.1 \mathrm{~nm}$.

To demonstrate the $\mathrm{CD}$ compensation algorithm, the signal of a commercially available $40 \mathrm{~Gb} / \mathrm{s}$ QPSK transmitter was routed through dispersion compensating fiber (DCF), yielding a net dispersion of $-4100 \mathrm{ps} / \mathrm{nm}$. The system correctly identified the modulation format as QPSK implemented with a DPMZ, the clock rate as $21.509 \mathrm{GHz}$, and the required dispersion compensation as $+4100 \mathrm{ps} / \mathrm{nm}$. Fig. 7 shows the constellation of the received signal before (a) and after (b) the digital dispersion compensation is applied. It is evident from the well-corrected constellation that both the clock rate and the dispersion are correctly identified. The principal distinguishing characteristic of a QPSK DPMZ signal-a 4-spot constellation with direct transitions between all symbol pairs-is likewise evident.

To illustrate the flexibility of the FID system in a WDM environment, it was tested against a signal consisting of 19 channels with a variety of formats selected from Table I. To model the characteristics of a mixed-signal, optically-routed network, the modulation clock rates were chosen to cover a 10-fold range of data rates, and a variety of dispersions were applied to impair the signals. Fig. 8 illustrates the spectrum of the channel plan. Four channels $(1,3,14,19)$ were modulated with a BPSK RZ33 transmitter at $10.709 \mathrm{~Gb} / \mathrm{s}$; these were passed through DCF with a net dispersion of -2500 to $-2800 \mathrm{ps} / \mathrm{nm}$, depending on wavelength. Three channels $(2,15,18)$ were modulated with an OOK RZ67 transmitter at $10.709 \mathrm{~Gb} / \mathrm{s}$ and were passed through DCF
TABLE II

FORMAT IDENTIFICATION RESULTS

\begin{tabular}{|ccccc|}
\hline$\#$ & $\begin{array}{c}\lambda \\
(\mathrm{nm})\end{array}$ & $\begin{array}{c}\text { Rate } \\
(\mathrm{GHz})\end{array}$ & Format & $\begin{array}{c}\text { Comp. } \\
(\mathrm{ps} / \mathrm{nm})\end{array}$ \\
\hline 1 & 1531.132 & 10.709 & BPSK RZ33 & 2500 \\
2 & 1531.524 & 10.709 & OOK RZ67 & 1350 \\
3 & 1531.909 & 10.709 & BPSK RZ33 & 2500 \\
4 & 1538.177 & 9.953 & OOK NRZ & -400 \\
5 & 1540.567 & 21.328 & OOK NRZ & -800 \\
6 & 1541.363 & 4.250 & OOK NRZ & 950 \\
7 & 1542.952 & 4.250 & OOK NRZ & 950 \\
8 & 1544.541 & 4.250 & OOK NRZ & 950 \\
9 & 1546.121 & 4.250 & OOK NRZ & 950 \\
10 & 1547.733 & 21.328 & QPSK DPMZ & -50 \\
11 & 1548.525 & 21.328 & OOK NRZ & -850 \\
12 & 1552.508 & 21.328 & QPSK DPMZ & -50 \\
13 & 1554.103 & 21.328 & QPSK DPMZ & 0 \\
14 & 1554.539 & 10.709 & BPSK RZ33 & 2700 \\
15 & 1555.345 & 10.709 & OOK RZ67 & 1500 \\
16 & 1555.752 & 21.328 & QPSK DPMZ & -50 \\
17 & 1556.527 & 21.328 & OOK NRZ & -850 \\
18 & 1557.363 & 10.709 & OOK RZ67 & 1500 \\
19 & 1558.582 & 10.709 & BPSK RZ33 & 2750 \\
\hline
\end{tabular}

with a net dispersion of -1300 to $-1500 \mathrm{ps} / \mathrm{nm}$. Eight channels were encoded with OOK NRZ signals at data rates of 9.953 (4), $21.328(5,11,17)$, and 4.25 (6-9) Gb/s. Channels 10, 12, 13 , and 16 were modulated with a QPSK DPMZ transmitter at 21.328 Gbaud. Channels 5, 11, and 17 were passed through $47 \mathrm{~km}$ of single mode fiber (SMF) yielding dispersion in the range of 750 to $825 \mathrm{ps} / \mathrm{nm}$. Note that the DCF and SMF both have dispersion slope-negative in the case of the former, and positive for the latter-so that the exact dispersion is wavelength dependent. The quoted ranges correspond to the nominal specifications for the fiber types used, for the wavelength range of 1530-1560 nm. No dispersion was applied to channels 4, 6$9,10,12,13$, and 16. For accuracy, the CD search grid used $50 \mathrm{ps} / \mathrm{nm}$ spacing irrespective of clock rate. While most channels were selected to be on $100 \mathrm{GHz}$ ITU grid wavelengths to match available WDM multiplexers, channels 1-3 and channels 15 and 16 were on a $50 \mathrm{GHz}$ channel grid to show system flexibility with respect to wavelength plan and also the capability of resolving channels that are not isolated in wavelength. OSNR values range from 13 to $>30 \mathrm{~dB} \cdot 0.1 \mathrm{~nm}$.

The results of processing the WDM channels are given in Table II. The baud rate is accurately identified for all channels to within $1 \mathrm{MHz}$. The optical modulation formats are likewise correctly identified. With two exceptions, the dispersion compensation is correctly identified to within $\pm 50 \mathrm{ps} / \mathrm{nm}$. Channel 4 is identified as requiring $-400 \mathrm{ps} / \mathrm{nm}$ of compensation, a result of its transmitter using a directly modulated laser. Channels 6-9 are identified as requiring dispersion compensation of $-950 \mathrm{ps} / \mathrm{nm}$. This result was caused by chirp from an unbalanced modulator in the transmitter. Note that in both cases, the system reports the effective dispersion compensation required to maximize the eye opening of these channels.

\section{DISCUSSION}

We have described an algorithm and experimentally demonstrated a system for identifying the optical modulation format, 
clock rate, and $\mathrm{CD}$ of a received optical signal with no a priori knowledge. We extensively validated this system with test cases comprising 14 modulation formats, OSNRs of 9 to 30 $\mathrm{db} \cdot 0.1 \mathrm{~nm}$, and dispersions of -4100 to $+1500 \mathrm{ps} / \mathrm{nm}$. We have also demonstrated the flexibility of our system with respect to wavelength, modulation format, and data rate using both selfbuilt and commercially available transmitters in a WDM system. The data rate, modulation format, and required dispersion compensation returned by our system provides the necessary information to configure a universal optical receiver with data demodulation algorithms for optimal filtering and dispersion compensation. It further demonstrates on-the-fly receiver reconfiguration to accommodate architecture changes such as rerouting signals in a mesh network, or reconfiguring transmitters to optimize network bandwidth utilization will be possible.

The technology concept demonstrated herein enables a CON architecture in which automatic reconfiguration of receivers is cued according to the identified signal formats and transport channel conditions. However, modulation format technology has advanced rapidly in recent years, so that implementation of a $\mathrm{CON}$ architecture using on-the-fly receiver reconfiguration will require extension of our approach to accommodate these advances, including polarization multiplexed modulation schemes [20], quadrature amplitude modulation formats [21], and potentially subcarrier multiplexing or superchannel schemes [22]. We have performed limited testing of polarization multiplexed signals using commercial transmitters with BPSK-MZ and QPSK-DPMZ formats (identical formats on each polarization tributary) with the FID algorithm, and confirmed that the signals were accurately identified. For these two test cases the format was correctly identified without polarization demultiplexing. These tests are not included in our results as the range of test parameters for these transmitters was very limited, compared to the extensive parameter space examined for the formats presented. General techniques for handling advanced modulation formats are under development. For polarization demultiplexing our approach is similar to that of Szafraniec [23]. To identify quadrature amplitude modulation formats we are evaluating techniques to perform constellation identification via pattern matching after symbol timing recovery. These new techniques are still in early development and will be discussed in future work.

\section{ACRONYMS}

$\begin{array}{ll}\text { AAM } & \text { auxiliary amplitude modulation } \\ \text { ADC } & \text { analog-to-digital converter } \\ \text { BPSK } & \text { binary phase-shift keying } \\ \text { CD } & \text { chromatic dispersion } \\ \text { CON } & \text { cognitive optical network } \\ \text { DCF } & \text { dispersion compensating fiber } \\ \text { DPMZ } & \text { dual-parallel Mach-Zehnder modulator } \\ \text { DSP } & \text { digital signal processing } \\ \text { FFT } & \text { fast-Fourier transform } \\ \text { FID } & \text { format identification algorithm } \\ \text { FOM } & \text { figure-of-merit } \\ \text { LO } & \text { local oscillator } \\ \text { MZM } & \text { Mach-Zehnder modulator }\end{array}$

MZPM Mach-Zehnder followed by a phase modulator

NRZ non return-to-zero

OCM optical channel monitor

ODB Optical-duo-binary

OOK on-off keying

OSNR optical signal-to-noise ratio

PF programmable optical filter

PM phase modulator

PSK phase-shift keying

QPSK quarternary phase-shift keying

SOP state of polarization

WDM wavelength-division multiplexing

\section{REFERENCES}

[1] M. G. Taylor, "Coherent detection method using DSP for demodulation of signal and subsequent equalization of propagation impairments," IEEE Photon. Technol. Lett., vol. 16, no. 2, pp. 674-676, Feb. 2004.

[2] K. Roberts, M. O’Sullivan, K.-T. Wu, H. Sun, A. Awadalla, D. J. Krause, and C. Laperle, "Performance of dual-polarization QPSK for optical transport systems," J. Lightw. Technol., vol. 27, no. 16, pp. 3546-3559, Aug. 2009.

[3] K. Kikuchi, "Phase-diversity homodyne detection of multilevel optical modulation with digital carrier phase estimation," IEEE J. Sel. Topics Quantum Electron., vol. 12, no. 4, pp. 563-570, Aug. 2006.

[4] S. J. Savory, "Digital filters for coherent optical receivers," Opt. Exp., vol. 16, no. 2, pp. 804-817, 2008.

[5] X. Li, X. Chen, G. Goldfarb, E. Mateo, I. Kim, F. Yaman, and G. Li, "Electronic post-compensation of WDM transmission impairments using coherent detection and digital signal processing," Opt. Exp., vol. 16, no. 2, pp. 880-888, 2008.

[6] E. M. Ip and J. M. Kahn, "Fiber impairment compensation using coherent detection and digital signal processing," J. Lightw. Technol., vol. 28, no. 4, pp. 502-519, Feb. 2010.

[7] X. Zhou and J. Yu, "Multi-level, multi-dimensional coding for high-speed and high-spectral-efficiency optical transmission," J. Lightw. Technol., vol. 27, no. 16, pp. 3641-3653, Aug. 2009.

[8] O. A. Dobre, A. Abdi, Y. Bar-Ness, and W. Su, "Survey of automatic modulation classification techniques: Classical approaches and new trends," IET Commun., vol. 1, no. 2, pp. 137-156, 2007

[9] W. Wei, C. Wang, and J. Yu, "Cognitive optical networks: Key drivers, enabling techniques, and adaptive bandwidth services," IEEE Commun. Mag., vol. 50, no. 1, pp. 106-113, Jan. 2012.

[10] J. Mitola and G. Q. Maguire, "Cognitive radio: Making software radios more personal," IEEE Pers. Commun., vol. 6, no. 4, pp. 13-18, Aug. 1999.

[11] P. J. Winzer and R. J. Essiambre, "Advanced Optical Modulation Formats," Proc. IEEE, vol. 94, no. 5, pp. 952-985, May 2006.

[12] W. Shieh and I. Djordjevic, OFDM for Optical Communications, 1st ed. London, U.K.: Academic, Oct. 2009.

[13] R. Schmogrow, D. Hillerkuss, M. Dreschmann, M. Huebner, M. Winter J. Meyer, B. Nebendahl, C. Koos, J. Becker, W. Freude, and J. Leuthold, "Real-time software-defined multiformat transmitter generating 64QAM at 28 GBd," IEEE Photon. Technol. Lett., vol. 22, no. 21, pp. 1601-1603, Nov. 2010

[14] W. Freude, R. Schmogrow, B. Nebendahl, D. Hillerkuss, J. Meyer, M. Dreschmann, M. Huebner, J. Becker, C. Koos, and J. Leuthold, "Software-defined optical transmission," in Proc. IEEE 13th Int. Conf. Transp. Opt. Netw., Aug. 2011, pp. 1-4.

[15] J. Zweck and C. Menyuk, "A chromatic dispersion estimation method for arbitrary modulation formats," in CLEO:2011 Laser Applications to Photonic Applications, OSA Technical Digest (CD) (Optical Society of America, 2011), paper CThX4. Available: http://www.opticsinfobase.org/abstract.cfm?URI=CLEO_SI-2011CThX

[16] Z. Pan, C. Yu, and A. E. Willner, "Optical performance monitoring for the next generation optical communication networks," Opt. Fiber Technol., vol. 16, no. 1, pp. 20-45, Jan. 2010.

[17] T. Clark and M. Dennis, "Coherent optical phase-modulation link," IEEE Photon. Technol. Lett., vol. 19, no. 16, pp. 1206-1208, Aug. 2007.

[18] G. Agrawal, Fiber Optic Communications. New York, NY, USA: Wiley, 2002. 
[19] M. Gasior and J. L. Gonzalez, "Improving FFT frequency measurement resolution by parabolic and Gaussian spectrum interpolation," in Proc. 11th BEAM Instrum. Worksh., Nov. 2004, pp. 276-285.

[20] E. Ip, A. P. T. Lau, D. J. F. Barros, and J. M. Kahn, "Coherent detection in optical fiber systems," Opt. Exp., vol. 16, no. 2, pp. 753-791, 2008.

[21] P. J. Winzer, A. H. Gnauck, C. R. Doerr, M. Magarini, and L. L. Buhl, "Spectrally efficient long-haul optical networking using 112-Gb/s polarization-multiplexed 16-QAM," J. Lightw. Technol., vol. 28, no. 4, pp. 547-556, Feb. 2010.

[22] S. Chandrasekhar and X. Liu, "Terabit superchannels for high spectral efficiency transmission," in Proc. IEEE 36th Eur. Conf. Exhib. Opt. Commun., 2010, pp. 1-6.

[23] B. Szafraniec, B. Nebendahl, and T. Marshall, "Polarization demultiplexing in stokes space," Opt. Exp., vol. 18, no. 17, pp. 17 928-17 939, 2010.

Eric J. Adles (M'03) received the B.S. and Ph.D. degrees in physics from North Carolina State University, Raleigh, NC, USA. His graduate research focused on the application of second-harmonic generation for real-time optical diagnostics of semiconductors. From 2009 to 2010, he was a Postdoctoral Researcher at the University of Maryland Baltimore County, Baltimore, MD, USA, where he worked on optoelectronic oscillators and photonic phase noise measurement systems. He is currently a Member of the Senior Professional Staff at the Johns Hopkins University Applied Physics Laboratory, Laurel, MD, USA, where he works on photonic systems for radio frequency and digital communication applications. Dr. Adles is a Member of the IEEE Photonics Society, the Optical Society of America, and the American Physical Society. He is currently serving in the Microwave Photonics Committee for the IEEE Photonics Conference.

Michael L. Dennis (M'92-SM'04) received the B.S. degree in physics from Western Kentucky University, Bowling Green, KY, USA, in 1984, the M.S. degree in physics and the $\mathrm{Ph} . \mathrm{D}$. degree in optical science, both from the University of New Mexico, Albuquerque, NM, USA, in 1988 and 1991, respectively. He is currently a Member of the Principal Professional Staff at the Applied Physics Laboratory (APL), Johns Hopkins University (JHU), Laurel, MD, USA, where he is developing photonic technologies for application to radio frequency and optical communications and sensor systems. From 1991 to 2000, he was a Research Physicist in the Optical Sciences Division, Naval Research Laboratory, Washington, DC, USA, where his research interests were in the fields of fiber lasers and optical communications. In 2000, he joined the venture-backed Dorsal Networks, Columbia, MD, later acquired by Corvis Corporation, Columbia, where he was a Senior Optical Engineer developing hardware for telecommunications applications, including undersea and terrestrial wavelength-division multiplexing transmission systems. From 2003 to 2004, he was a Telecommunications Design Engineer at the NASA Goddard Space Flight Center, Greenbelt, MD. In 2004, he joined APL, JHU, as a member of the Senior Professional Staff and was named to the Principal Professional Staff in 2009. His current research interests include development of low-noise and ultrafast fiber lasers, fiber optic and free space optical communications systems and the application of photonics microwaves, and millimeter wave systems. Dr. Dennis is a Member of the IEEE Photonics Society and the Optical Society of America, and is presently a Member of the Optical Processing and Analog Subsystems Technical Subcommittee for the Optical Fiber Communications Conference.

Wallace R. Johnson, biography not available at the time of publication.
Timothy P. McKenna (M'11) received the B.S. degree in electrical engineering from the University of Pennsylvania, Philadelphia, PA, USA, in 2009, and the M.S. degree in electrical engineering from Stanford University, Stanford, CA, USA, in 2011. He is currently an Associate Professional Staff member at the Johns Hopkins University Applied Physics Laboratory, Laurel, MD, USA. His current research includes high data rate millimeter wave wireless communications, photonics-assisted analog-to-digital conversion, and next generation optical fiber communications systems.

Curtis R. Menyuk was born March 26, 1954. He received the B.S. and M.S. degrees from the Massachusetts Institute of Technology, Cambridge, MA, USA, in 1976, and the Ph.D. degree from University of California, Los Angeles, Los Angeles, CA, USA, in 1981. He has worked as a Research Associate at the University of Maryland, College Park, MD, USA, and at Science Applications International Corporation, McLean, VA, USA. In 1986, he became an Associate Professor in the Department of Electrical Engineering, University of Maryland Baltimore County (UMBC), Baltimore, MD, USA, and he was the founding member of this department. In 1993, he was promoted to Professor. He was on partial leave from UMBC from Fall, 1996 until Fall, 2002. From 1996-2001, he worked part-time for the Department of Defense, co-directing the Optical Networking Program at the DoD Laboratory for Telecommunications Sciences in Adelphi, MD, USA, from 1999-2001. In 2001-2002, he was the Chief Scientist at PhotonEx Corporation. In 2008-2009, he was a JILA Visiting Fellow at the University of Colorado. For the last 25 years, his primary research area has been theoretical and computational studies of lasers, nonlinear optics, and fiber optic communications. He has authored or coauthored more than 240 archival journal publications as well as numerous other publications and presentations, and he is a coinventor of five patents. He has also edited three books. The equations and algorithms that he and his research group at UMBC have developed to model optical fiber systems are used extensively in the telecommunications and photonics industry. Dr. Menyuk is a Member of the Society for Industrial and Applied Mathematics and of SPIE. He is a fellow of the American Physical Society and the Optical Society of America. He is a former UMBC Presidential Research Professor, and he received the 2013 IEEE Photonics Society William Striefer Award.

Joseph E. Sluz received the B.S.E.E. degree from the University of Illinois/Champaign, USA and the M.S.E.E. degree from The Johns Hopkins University Applied Physics Laboratory, MD, USA. He is currently a Principal Professional Staff Member at the Johns Hopkins University Applied Physics Laboratory. He has more than 20 years of experience in radio frequency and microwave design, including high-speed fiber optic telecommunications hardware. He draws upon his expertise acquired through numerous years of development work on radar and communications electronics for government and defense applications at Northrop Grumman and APL as well as electro-optic systems for telecommunications at Yafo Networks Inc. He has particularly relevant prior experience in the design of 10 and $40 \mathrm{Gbps}$ fiber optic transmission and receiving systems and the development of optical polarization mode dispersion compensators using a variety of transmission formats and data rates.

Raymond M. Sova (M'95) received the B.S. degree in electrical engineering from Pennsylvania State University, State College, PA, USA, in 1986, the M.S. degree in applied physics from the Johns Hopkins University, Laurel, MD, USA, in 1990, and the Ph.D. degree in electrical engineering from the Johns Hopkins University, Baltimore, MD, in 2003. He is currently a Member of the Principal Professional Staff with the Johns Hopkins University Applied Physics Laboratory, Laurel, and an Assistant Research Professor at the Johns Hopkins University, Baltimore. He has authored more than 40 technical papers. His areas of research include nonlinear optics, fiber-optic communication, RF photonics, and laser remote sensing. 
Michael G. Taylor (M'94) received the B.A. degree in physics from Oxford University, Oxford, U.K., in 1987, and the Ph.D. degree in electrical and electronic engineering from University College London, London, U.K., in 1990. His doctoral thesis concerned multiquantum-well modulator devices for optical computing applications. He joined STC Technology Ltd. (subsequently Nortel), Harlow, U.K., in 1990, to work on the development of optical fiber communications systems using EDFAs. He contributed to the design of the first transoceanic transmission systems using optical amplifiers, and worked on early research projects in 1550 band WDM and the NTON all-optical network demonstration. In 1996, he joined Ciena Corporation, MD, USA. He worked on the design of several of that company's dense WDM products, including the $10-\mathrm{Gb} / \mathrm{s}$ product, and in management of fiber propagation effects in general. Since 2002, he has been an independent consultant and has conducted research on coherent optical communications in collaboration with groups at University College London and University of Central Florida, Orlando, FL, USA. He has published more than 30 research papers and has been granted 17 US patents. Dr. Taylor is a Regular Reviewer for the IEEE Photonics Technology Letters and the Journal of Lightwave Technology.
Radha A. Venkat (M'11) received the B.S. degree in applied physics from Case Western Reserve University, Cleveland, OH, USA, in 1996, and the M.S.E. degree in electrical engineering from Princeton University, Princeton, NJ, USA, in 1998. She is currently a Senior Professional Staff Member at the Johns Hopkins University Applied Physics Laboratory, Laurel, MD, USA, in the Optical Communications Systems section where she works in free-space and fiber optic communications. From 2003 to 2008, she was a Senior Member of Engineering Staff at Lockheed Martin MS2, Moorestown, NJ, USA, where she worked variously in Weapons Control, Simulation \& Modeling, and Command \& Decision as a Systems Engineer and Analyst. From 1998 to 2003, she was a Member of Technical Staff at Lucent Technologies Specialty Fiber Devices, Somerset, NJ, where she worked in erbium fiber characterization and fiber optic component design. 